\title{
1 Molecular signature and target-specificity of inhibitory circuits formed by 2 Martinotti cells in the mouse barrel cortex
}

3 Cristina Donato ${ }^{1,2}$, Carolina Cabezas ${ }^{1}$, Andrea Aguirre ${ }^{1}$, Joana Lourenço ${ }^{1}$, Marie-Claude Potier ${ }^{1}$, Javier

4 Zorrilla de San Martin ${ }^{1 *}$, Alberto Bacci ${ }^{1 *}$

5

6 'Sorbonne Université, Institut du Cerveau - Paris Brain Institute - ICM, Inserm, CNRS, APHP, Hôpital de 7 la Pitié Salpêtrière, Paris, France

8 2Present address: Luxembourg Centre for Systems Biomedicine, University of Luxembourg, Belvaux, $912 \mathrm{~L}-4367$, Luxembourg.

*Corresponding authors: alberto.bacci@icm-institute.org; javier.zorrilla@icm-institute.org

Abbreviated title (50-character maximum): Martinotti-mediated target-dependent inhibition.

Number of pages: 37

Number of Figures: 6

Number of tables: 0

Conflict of Interest: The authors declare no competing financial interests.

Acknowledgments: We thank the ICM technical facilities PHENO-ICMICE, iGENSEQ and ICM.Quant. This work was supported by "Investissements d'avenir" ANR-10-IAIHU-06, BBT-MOCONET1; BBTMOCONET2; Agence Nationale de la Recherche (ANR-13-BSV4-0015-01 ; ANR-16-CE16-0007-02 ; ANR17-CE16-0026-01 ; ANR-18-CE16-0011-01 ; ANR-20-CE16-0011-01; ANR-12-EMMA-0010;), Fondation Recherche Médicale (Equipe FRM DEQ20150331684 and EQU201903007860), NARSAD independent Investigator Grant and Fondation Lejeune (\#1790). CD was supported by the École de Neurosciences de Paris et île de France (ENP) and by the Labex Bio-Psy. PHENO-ICMICE was supported by two "Investissements d'avenir" (ANR-10-IAIHU-06 and ANR-11-INBS-0011-NeurATRIS) and the "Fondation pour la Recherche Médicale". 


\section{Abstract}

In the neocortex, fast synaptic inhibition orchestrates both spontaneous and sensory-evoked activity. GABAergic interneurons (INs) inhibit pyramidal neurons (PNs) directly, modulating their output activity and thus contributing to balance cortical networks. Moreover, several IN subtypes also inhibit other INs, forming specific disinhibitory circuits, which play crucial roles in several cognitive functions. Here, we studied a homogeneous subpopulation of somatostatin (SST)-positive INs, the Martinotti cells (MCs) in layer $2 / 3$ of the mouse barrel cortex (both sexes). MCs are a prominent IN subclass inhibiting the distal portion of PN apical dendrites, thus controlling dendrite electrogenesis and synaptic integration. Yet, it is poorly understood whether MCs inhibit other elements of the cortical circuits, and the connectivity properties with non-PN targets are unknown. We found that MCs have a strong preference for PN dendrites, but they also considerably connect with parvalbumin (PV)-positive, vasoactive intestinal peptide (VIP)-expressing and layer 1 (L1) INs. Remarkably, GABAergic synapses from MCs exhibited clear cell-type-specific short-term plasticity. Moreover, whereas the biophysical properties of MC-PN synapses were consistent with distal dendritic inhibition, MC-IN synapses exhibited characteristics of fast perisomatic inhibition. Finally, MC-PN connections used $\alpha 5$-containing $\mathrm{GABA}_{\mathrm{A}} \mathrm{Rs}$, but this subunit was not expressed by the other INs targeted by MCs. We reveal a specialized connectivity blueprint of MCs within different elements of superficial cortical layers. In addition, our results identify $\alpha 5-G A B A_{A} R s$ as the molecular fingerprint of MC-PN dendritic inhibition. This is of critical importance, given the role of $\alpha 5-G A B A_{A} R s$ in cognitive performance and their involvement in several brain diseases.

\section{Significance statement}

Martinotti cells (MCs) are a prominent subclass of SST-expressing GABAergic INs, specialized in controlling distal dendrites of PNs and taking part in several cognitive functions. Here we characterize the connectivity pattern of MCs with other INs in the superficial layers (L1 and L2/3) of the mouse 
56 barrel cortex. We found that the connectivity pattern of MCs with PNs as well as PV, VIP and L1 INs

57 exhibit target-specific plasticity and biophysical properties. The stark specificity of $\alpha 5-G A B A_{A} R s$ at MC-

58 PN synapses, and the lack or functional expression of this subunit by other cell types, define the molecular identity of MC-PN connections and the exclusive involvement of this outstanding inhibitory circuits in $\alpha 5$-dependent cognitive tasks.

61 


\section{Introduction}

In the neocortex, fast synaptic inhibition underlie important cognitive-relevant activity (Buzsáki, 2010; Isaacson and Scanziani, 2011). Neocortical inhibition is provided by GABAergic interneurons, which are highly heterogeneous and connect with both principal pyramidal neurons (PNs) and other inhibitory cells in a very stereotyped manner. Some interneurons, such as parvalbumin (PV)expressing basket cells, innervate the perisomatic region of cortical PNs, and they thus provide a tight temporal control of PN spiking output and drive cognition-relevant fast network oscillations, especially in the $\beta$ - $\gamma$-frequency range (20-100 Hz)(Bartos et al., 2007; Buzsáki and Wang, 2012).

Conversely, other interneuron types, such as those expressing the neuropeptide somatostatin (SST), were shown to target dendrites of PNs, thereby controlling dendritic electrogenesis, non-linear integration and glutamatergic synaptic input (Wang et al., 2004; Lovett-Barron et al., 2012; Wilson et al., 2012; Schulz et al., 2018). In sensory cortices, SST interneurons were shown to be involved in lateral inhibition, playing a major role in key sensory computations, such as surround suppression (Kapfer et al., 2007; Silberberg and Markram, 2007; Berger et al., 2009; Adesnik and Scanziani, 2010; Adesnik et al., 2012). Moreover, SST-operated dendritic inhibition was shown to encode fear memory and affective behavior in prefrontal cortex (Xu et al., 2013; Scheggia et al., 2019; Clem and Cummings, 2020).

SST INs were proposed to be the source of a profuse 'blanket' of inhibition due to their dense connectivity with PNs (Fino and Yuste, 2011). However, this view neglects the diversity of SST-positive INs (Gouwens et al., 2020), and the fact that they preferentially contact specific PN subclasses (Hilscher et al., 2016) as well as other inhibitory neurons (Pfeffer et al., 2013; Tremblay et al., 2016). In particular, SST interneurons can be classified as Martinotti cells (MCs) and non-Martinotti cells, which exhibit differential connectivity patterns as well as specific molecular profiles (Wang et al., 2004; Ma et al., 2006; Tremblay et al., 2016; Yavorska and Wehr, 2016; Paul et al., 2017; Scala et al., 2019). In particular, MCs exhibit a well-defined axonal morphology, as they project their axons to layer 1, 
where they extensively inhibit the most distal dendritic tufts of PNs (Wang et al., 2004; Ma et al., 2006; Kapfer et al., 2007; Silberberg and Markram, 2007; Tremblay et al., 2016). Functionally, MCs are efficiently recruited by local PNs with loose-coupled, strongly facilitating synapses (Reyes et al., 1998; Wang et al., 2004; Kapfer et al., 2007; Silberberg and Markram, 2007), and are quasi-preferentially inhibited by vasoactive intestinal peptide (VIP)-expressing GABAergic interneurons (Pfeffer et al., 2013; Karnani et al., 2016; Tremblay et al., 2016; Walker et al., 2016). Finally, MCs form synapses with other elements of the cortical circuit, namely other inhibitory interneurons (Ma et al., 2006; Pfeffer et al., 2013). However, the actual extent and biophysical properties of these disinhibitory circuits are unknown and/or generalized over SST-expressing MCs and nMCs (Pfeffer et al., 2013).

Importantly, MC-PN inhibitory synapses were shown to use the $\alpha 5$-containing $G A B A_{A} R$ ( $\alpha 5$ $\mathrm{GABA}_{A} \mathrm{Rs}$ ) (Ali and Thomson, 2008; Zorrilla de San Martin et al., 2020). Similarly, the hippocampal counterparts of MCs, the oriens-lacunoso moleculare (O-LM) interneurons express functional $\alpha 5$ $\mathrm{GABA}_{A} \mathrm{Rs}$ (Schulz et al., 2018). This prompts the question whether GABAergic synapses formed by MCs onto other elements of the cortical circuit use this specific subunit of $G A B A_{A} R s$. Understanding the actual synaptic circuits relying on the $\alpha 5$ subunit has important clinical implications. Indeed, $\alpha 5$ $\mathrm{GABA}_{A} \mathrm{Rs}$ were indicated as a prominent target for therapeutic interventions for cognitive dysfunctions in Down syndrome (Braudeau et al., 2011; Duchon et al., 2019; Schulz et al., 2019; Zorrilla de San Martin et al., 2020), depression (Zanos et al., 2017), anesthesia-induced memory impairment (Zurek et al., 2014) and schizophrenia (Duncan et al., 2010; Gill and Grace, 2014).

Here we investigated the connectivity blueprint of MCs in the superficial layers of the mouse barrel cortex. We found that, in addition to the known connectivity with PN distal dendrites, MCs connect extensively also with PV, VIP and L1 INs, but not with other MCs. Interestingly, GABAergic synapses formed by MCs exhibited clear target specificity of short-term plasticity. Finally, dendritic inhibition using $\alpha 5-G A B A_{A} R s$ is a peculiarity of MC-PN synapses, as unitary responses from MCs to other INs exhibited fast $(<1 \mathrm{~ms})$ rise-time, and they were not modulated by a $\alpha 5$ negative allosteric modulator (NAM). 

MCs for inhibitory synapses that they make with PNs and other elements of the cortical circuit.

\section{Materials and Methods}

117

\section{Animals}

Experimental procedures followed national and European (2010/63/EU) guidelines and have been approved by the author's institutional review boards and national authorities (APAFIS \#2599). All efforts were made to minimize suffering and reduce the number of animals. Mice used in this study were of both sexes. In order to identify GABAergic transmission from different INs we used several mouse models. To record from PV INs we initially used Pvalb-cre mice (Jackson Laboratory, Stock Number: 008069), crossed with a mouse line, which expresses a loxP-flanked STOP cassette and giving robust tdTomato fluorescence following Cre-mediated recombination (Jackson Laboratory Stock Number 007909). In the experiments illustrated in Figs. 2,3,5 and 6, we used PValbTomato mouse line (Kaiser et al., 2016, Jackson Stock\# 27395), a line that expresses TdTomato fluorescent protein specifically in PV INs. To record from MCs, we used GAD-67 GFP X98 mice (Ma et al., 2006), herein defined as X98. These mice express EGFP in a specific subset of GABAergic cells (Jackson Laboratory Stock\# 006340). To perform simultaneous recordings from MCs and PV INs we crossed X98 mice with PvAlb-tdtomato. Furthermore, in order to record from synaptically connected VIP INs and MCs we crossed VIP-Cre mice (Jackson Laboratory Stock \#010908) with X98 mice and infected newborns with viral vectors carrying the genes of either ChR2 or TdTomato (see below details of different viral infections).

\section{In Vitro Slice Preparation and Electrophysiology}

Coronal slices (300-350 $\mu \mathrm{m}$ thick) from somatosensory cortex were obtained from 18- to 25 -d-old mice. Animals were deeply anesthetized with isoflurane and decapitated. Brains were quickly 
removed and immersed in "cutting" solution $\left(4^{\circ} \mathrm{C}\right)$ containing the following (in $\mathrm{mM}$ ): 126 choline, 11 glucose, $26 \mathrm{NaHCO}_{3}, 2.5 \mathrm{KCl}, 1.25 \mathrm{NaH}_{2} \mathrm{PO}_{4}, 7 \mathrm{MgSO}_{4}$ and $0.5 \mathrm{CaCl}_{2}$ (equilibrated with 95-5\% $\mathrm{O}_{2}-\mathrm{CO}_{2}$, respectively). Slices were cut with a vibratome (Leica) in the same cutting solution and then incubated in oxygenated artificial cerebrospinal fluid (aCSF) containing the following (in $\mathrm{mM}$ ): $126 \mathrm{NaCl}, 2.5 \mathrm{KCl}$, $2 \mathrm{CaCl}_{2}, 1 \mathrm{MgSO}_{4}, 1.25 \mathrm{mM} \mathrm{NaH}_{2} \mathrm{PO}_{4}, 26 \mathrm{mM} \mathrm{NaHCO}_{3}$, and $16 \mathrm{mM}$ glucose (pH 7.4), initially at $34^{\circ} \mathrm{C}$ for $30 \mathrm{~min}$, and subsequently at room temperature until transfer to the recording chamber. Recordings were obtained at $32-34^{\circ} \mathrm{C}$. Whole-cell voltage-clamp recordings were performed in from layer (L)2/3 PNs, MCs, PV, VIP INs and L1 INs of the primary somatosensory cortex. PNs were visually identified using infrared video microscopy by their large somata and pia-oriented apical dendrites. L1 INs were also visually identified with transmitted light only as they are the only cell type with the soma present in L1. MCs (labeled with GFP, see Fig 1), VIP INs and PV INs (labeled with TdTomato), were identified using LED illumination (blue, $\lambda=470 \mathrm{~nm}$, green $\lambda=530 \mathrm{~nm}$, OptoLED system, Cairn Research, Faversham, UK) coupled to epifluorescent optical pathway of the microscope. Single or double voltage-clamp whole-cell recordings were made with borosilicate glass capillaries (with a tip resistance of 2-4 MS) filled with different intracellular solutions depending of the experiment. For unitary inhibitory postsynaptic currents (uIPSCs) the intracellular solution contained (in $\mathrm{mM}$ ): $70 \mathrm{~K}$-gluconate, $70 \mathrm{KCl}, 10$ at the end in some experiments. For tonic inhibition experiments, GABA (5 $\mu \mathrm{M})$ was added to the aCSF. To record unitary excitatory postsynaptic currents (uEPSCs) from INs, a low chloride intracellular solution was used and DNQX was omitted in the aCSF superfusate. In these experiments, the intracellular solution had the following composition (in $\mathrm{mM}$ ): $150 \mathrm{~K}$-gluconate, $4.6 \mathrm{MgCl}_{2}, 10 \mathrm{HEPES}, 1$ EGTA, $0.1 \mathrm{CaCl}_{2}$, 4 Na-ATP, $0.4 \mathrm{Na}-\mathrm{GTP}$, pH adjusted to 7.2 with $\mathrm{KOH}, 280-300 \mathrm{mOsm}$. In voltage-clamp experiments, access resistance was on average $<15 \mathrm{M} \Omega$ and monitored throughout the experiment. 
experiment. Unitary synaptic responses were elicited in voltage-clamp mode by brief somatic depolarizing. A train of 5 presynaptic spikes at $50 \mathrm{~Hz}$ was applied to infer short-term plasticity of synaptic responses. The paired pulse ratio (PPR) was obtained as the peak amplitude of the second uEPSC divided by that of the first. In order to isolate GABA $A_{A}$-receptor-mediated currents, DNQX (10 $\mu \mathrm{M})$ was present in the superfusate of all experiments, unless otherwise indicated. $10 \mathrm{KHz}$ (for current clamp experiments). All drugs were obtained from Tocris Cookson (Bristol, UK) or Sigma (Bristol, UK). $\alpha 5$ IA, (3-(5-methylisoxazol-3-yl)-6-[(1-methyl-1,2,3-triazol-4-yl)methyloxy]-1, 2, 4triazolo[3, 4-a]phthalazine) also named L-822179 was synthesized by Orga-Link SARL (Magny-lesHameaux, France) according to Sternfeld et al. (2004) as in Braudeau et al. (2011). The hydrochloride salt was solubilized in DMSO at a concentration of $1 \mathrm{mM}$ and then diluted in the appropriate buffer.

Virus-Mediated Gene Delivery and Optogenetics

To study MC-VIP and VIP-MC synapses we first crossed crossed VIPcre with X98 mice and injected $300 \mathrm{~nL}$ of a solution containing adeno-associated viral (AAV) particles into the somatosensory cortex of ice-anesthetized pups (P0-3) to selectively express TdTomato or Channelrhodopsin-2 (ChR2) in VIP INs. Injections were made with a beveled glass pipette $300 \mu \mathrm{m}$ deep in the somatosensory cortex through intact skin and skull. We then delivered the solution containing the AAVs using a Nanoliter 2000 Injector (WPI Inc., USA). The pipette was left in place for an additional $30 \mathrm{~s}$, before it was retracted. The AAVs expressed floxed ChR2 or TdTomato (AAV9.EF1.dflox.hChR2(H134R)mCherry.WPRE.hGH; Addgene \#20297 and pAAV-FLEX-tdTomato; Addgene \#28306, respectively) purchased from the Penn Vector Core (University of Pennsylvania). At the end of the procedure, pups were returned to their mother. ChR2 activation was obtained by brief (0.5-2 ms) LED light pulses on cortical slices $(\lambda=470 \mathrm{~nm})$. Experiments were performed using a $60 \mathrm{X}$ water immersion lens. Light- 
evoked responses were recorded in L 2/3 MCs and were completely abolished by gabazine (not shown).

\section{Data analysis}

Experiments on firing dynamics, tonic currents and unitary paired recordings were analyzed with routines (courtesy J. R. Huguenard, Stanford University). function:

$$
F(\mathrm{t})=A_{\text {fast }} e^{\frac{-t}{\tau_{\text {fast }}}}+A_{\text {slow }} e^{\frac{-t}{\tau_{\text {slow }}}}
$$

Equation (1)

where $A_{\text {fast }}$ and $A_{\text {slow }}$ are the fast and slow amplitude components, and $\tau_{\text {fast }}$ and $\tau_{\text {slow }}$ are the fast and slow decay time constants, respectively. The weighted decay time constant $\left(\tau_{\mathrm{d}, \mathrm{w}}\right)$ was calculated using the following equation:

$$
\tau_{\mathrm{d}, \mathrm{w}}=\frac{\left[\left(A_{\text {fast }} \cdot \tau_{\text {fast }}\right)+\left(A_{\text {slow }} \cdot \tau_{\text {slow }}\right)\right]}{A_{\text {fast }} \cdot A_{\text {slow }}}
$$

The adaptation index was calculated as the last/first inter-spike interval ratio following a train of spikes induced by injection of a depolarizing step of current. Passive properties as well as synaptic currents were analyzed with Clampfit and custom-made scripts in MATLAB (Mathworks). Both unitary and light-induced IPSCs were averaged across at least 20 sweeps for each condition examined. Results are presented as means \pm SEM unless otherwise stated. 

included in the intracellular solution at a high concentration $(10 \mathrm{mg} / \mathrm{mL})$, which required extensive sonication. To avoid excessive degradation of fragile molecules such as ATP, sonication was performed in an ice bath. The intracellular solution was then filtered twice to prevent the presence of undissolved lumps of biocytin in the patch pipette. Recordings lasted for at least $30 \mathrm{~min}$. During that time, access resistance was continuously monitored throughout the experiment. At the end of recordings, the patch pipette was removed carefully to obtain an outside-out patch in order to reseal the cell properly.

The slice was then left in the recording chamber for a further 5-10 min to allow biocytin diffusion.

Slices were then fixed with $4 \%$ paraformaldehyde in phosphate buffer saline (PBS, Sigma) for at least 48h. Following fixation, slices were incubated with the avidin-biotin complex (Vector Labs) and a high concentration of detergent (Triton-X100,5\%) for at least two days before staining with 3,3'Diaminobenzidine (DAB, AbCam). Cells were then reconstructed, and cortical layers delimited using Neurolucida (MBF Bioscience). Neuronal reconstructions were aligned to a mouse atlas from the Allen Institute. By using Neurolucida Explorer, we analyzed the length of axons and dendrites of MCs in L2/3 and L1 of somatosensory cortex. Data were exported and analyzed in OriginPro 2016 (OriginLab Corporation).

\section{Immunofluorescence}

Slices used for electrophysiology experiments were fixed overnight in $4 \%$ paraformaldehyde in phosphate buffered saline (PBS, $\mathrm{pH} 7.4$ ) at $4^{\circ} \mathrm{C}$. Slices were then rinsed three times at room temperature (10 min each time) in PBS and incubated overnight at $4^{\circ} \mathrm{C}$ in PB with $0.3 \%$ Triton X-1000, 0.1\% normal goat serum (NGS), anti-GFP antibody (host: rabbit, 1:400, AB3080, Millipore) and/or antiSST antibody (host: mouse, 1:250, G10 sc-55565, Santa Cruz) and/or anti-DsRed (host: rabbit, 1/500, \#632496 Takara Bio Clontech). Slices were then rinsed three times in PBS (10 min each) at room temperature and incubated with goat-hosted secondary antibodies coupled to different fluorophores: Alexa 488 (1:500, A11034, Life technologies) and Alexa 633 (1:500, A21052, Life technologies) for $2 \mathrm{~h}$ at room temperature. Slices were then rinsed three times in PBS (10 min each) at room temperature 
and mounted with Fluoromount. Immunofluorescence was then images were acquired with a confocal microscope (Leica SP8).

Parvalbumin, SST and GFP staining on X98 mice were also performed on $50 \mu \mathrm{m}$-thick slices. Briefly, mice were perfused with $0.9 \% \mathrm{NaCl}$ solution containing Heparin and $4 \%$ paraformaldehyde (PFA). Brains were cryo-protected by placing them overnight in $30 \%$ sucrose solution and then frozen in Isopentane at a temperature $<-50^{\circ} \mathrm{C}$. Brains were sliced with a freezing microtome (ThermoFisher and mouse anti-GFP antibody (1:500; Milipore MAB3580). Slices were then rinsed three times in PBS (10 min each) at room temperature and incubated with goat anti-rabbit and a goat anti-mouse antibody (1:500; Jackson IR) coupled to Alexa-488 or 633 for $3.5 \mathrm{hr}$ at room temperature. Slices were then rinsed three times in PBS (10 min each) at room temperature and coverslipped in mounting medium (Fluoromount, Sigma Aldrich F4680). Immunofluorescence images were acquired with a confocal (Leica SP8) or epifluorescence (Zeiss Apotome 3) microscope.

\section{Statistical Analysis}

All statistical analysis were performed in Origin (Microcal). Normality of the data was systematically assessed (Shapiro-Wilk normality test). Normal distributions were statistically compared using Paired $\mathrm{t}$-Test or Two-sample t-Test. When data distributions were not normal or $\mathrm{n}$ was small, non-parametric tests were performed (Mann-Whitney, Wilcoxon Signed Ranks Test). Two-way ANOVA tests were 


\section{Results}

260

261

262

263

264

265

266

267

268

269

270

271

272

273

274

275

276

277

278

\section{The X98 mouse is a reliable model to specifically study L2/3 Martinotti cells}

Despite being broadly classified as dendrite-targeting INs, SST-expressing cells exhibit significant electrophysiological, anatomical, connectivity and molecular heterogeneity (Ma et al., 2006; Paul et al., 2017; Naka et al., 2019). In order to specifically study the connectivity of L2/3 MCs we searched for a suitable mouse line. X98 mice express GFP predominantly in cortical layers (L) $5 B$ and 6 , and, to a lesser extent, in L2/3 (Ma et al., 2006). A detailed characterization of these mice showed that GFP is specifically expressed in L5 MCs (Ma et al., 2006). However, although there is prominent fluorescence in L2/3, GFP-expressing cells in this cortical layer were not analyzed. Therefore, we first set out to confirm that GFP-expressing cells in L2/3 belong to the specific SST-positive interneuron subtype defined as the MCs. We performed immunofluorescence staining on microtome-cut sections from X98 coronal somatosensory slices of 18-25-days-old mice and showed that all GFP-expressing cells also expressed SST while some SST-positive cells did not express GFP (Fig. 1 A). In another series of experiments, several GFP-expressing neurons were filled with biocytin during whole-cell recordings and their morphology was traced to assess somato-dendritic and axonal morphology. Axons of L2/3 GFP-expressing neurons were systematically oriented towards superficial layers and consistently reached L1 where they were profusely branched (red tracing in Fig. $1 \mathrm{~B}$ and C; $\mathrm{p}=4.4 \mathrm{e}^{-4}$, One-way ANOVA followed by Bonferroni post-hoc test, $F=7.4716, n=11$ reconstructed GFP-positive neurons). Conversely, GFP-expressing neurons dendrites were mostly located in L2/3 without reaching L1 (blue tracing in Fig. $1 \mathrm{~B}$ and C). We then assessed the excitability and passive properties of GFP-expressing neurons $(n=22)$ and compared their firing pattern with that of PV INs, the most abundant and perhaps best characterized GABAergic neuronal subtype (Fig $1 \mathrm{D}$ ). As previously described, the majority of the GFP-positive cells in X98 mice displayed a characteristic sag in response to hyperpolarizing current injection and a highly adapting firing behavior when depolarizing currents triggered repetitive spiking (Fig 1 D). Conversely, PV INs displayed fast-spiking, non-adapting pattern in response to depolarizing 
currents (adaptation index: $2.27 \pm 0.17$ and $1.07 \pm 0.04$ for GFP-expressing and PV INs, respectively; $p=1.1 e^{-5}$, Mann-Whitney $U$ test; Fig. $\left.1 \mathrm{D}\right)$, more hyperpolarized resting membrane potential $\left(V_{m}:-66 \pm\right.$ 1 and $-71 \pm 1 \mathrm{mV}$ for GFP-expressing and PV INs, respectively; $p=0.0017$, unpaired T test) and lower input resistance ( $R_{\mathrm{i}}: 189 \pm 11$ and $92 \pm 10 \mathrm{M} \Omega$ for GFP-expressing and PV INs, respectively; $p=8.1 \mathrm{e}^{-6}$, Mann-Whitney U test; Fig. 1 E). 

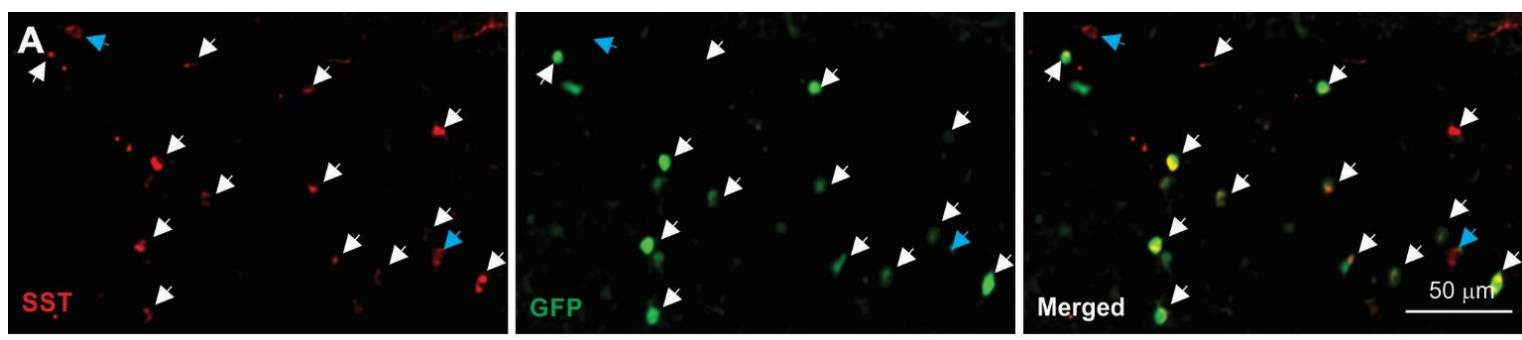

B

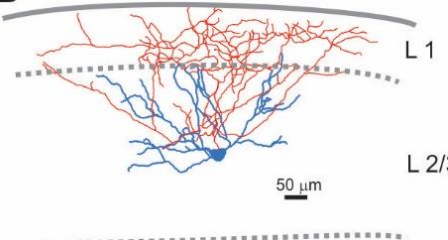

L $2 / 3$

D

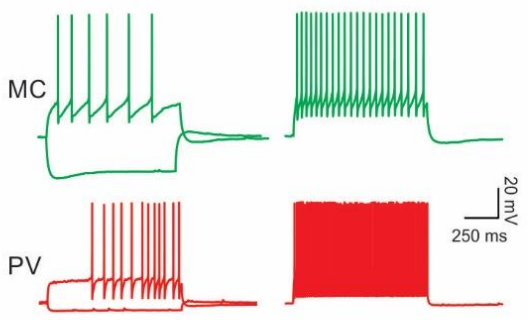

$\mathbf{F}$

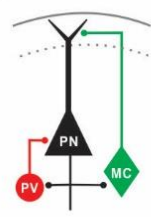

G

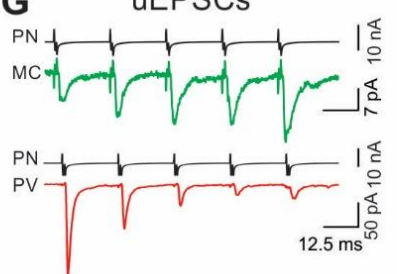

C
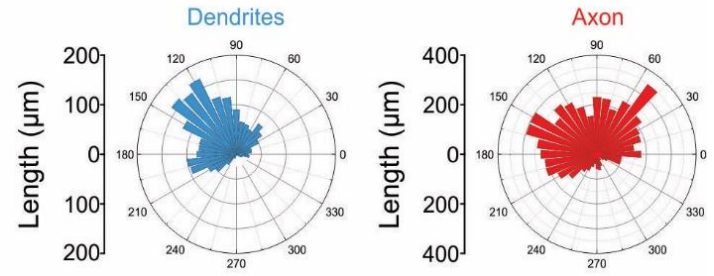

$\mathbf{E}$
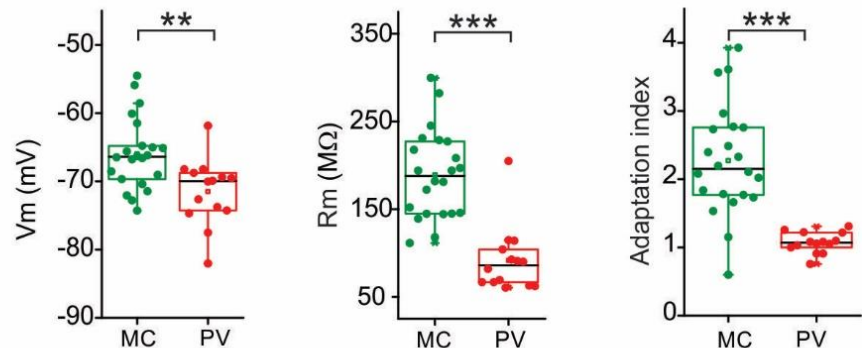

H

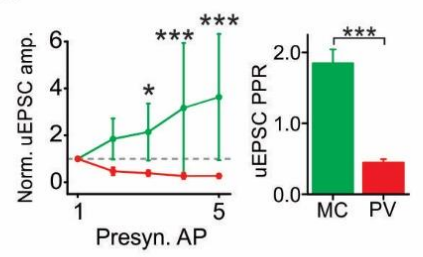

I ulPSCs
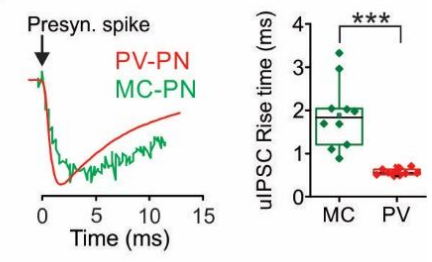

Figure 1: GFP-positive neurons in X98 mice are SST-expressing MCs.

A: Top: Epifluorescence micrograph of a dual immune-staining against SST (red) and GFP (green) in X98 coronal somatosensory slices. White arrows: GFP and SST co-localization; blue arrows: cells expressing SST only. B: Left: representative morphological reconstruction of a GFP-positive neuron filled with biocytin. Blue: dendrites; red: axons. Right: Population data of axon (red) and dendrite (blue) lengths distribution in L1 and L2/3 ( $n=11)$. C: Axonal (red) and dendritic polar plots of the cell of B. D: Representative current-clamp recordings from a GFP-expressing interneuron in X98 mice (green) and a PV cell (red). X98 GFP cells display a characteristic sag in response to hyperpolarizing current injection and a highly adapting firing behavior. Conversely, PV-cells show fast-spiking patterns in response to depolarizing current injections. E: Summary graphs of resting membrane potential (left), membrane resistance (middle) and adaptation index (right) in PV interneurons $(n=14)$ and MCs $(n=22)$. F: Schematic of mutually connected MC-PN and PV-PN pairs. G: Representative averaged voltage clamp trace of unitary EPSCs stimulated by 5 action potentials at $40 \mathrm{~Hz}$ in a PN and recorded in a GFP-positive cell from a X98 mouse (green, upper panel), and in a PV-cell (bottom panel). H: Left panel: pooled normalized amplitudes of uEPSC evoked with a $50 \mathrm{~Hz}, 5 \mathrm{AP}$ train. Right: population plot of paired-pulse ratio (PPR) of X98 GFP ( $n=20$, green) and PV-INs ( $n=11$, red). I: Left: overlapped representative uIPSCs elicited by MCs (green) and PV-INs (red) recorded PNs. Right: population plot of the uIPSC mean rise time from MC to PN (green) and PV to PN (red) synapses. ${ }^{*} p<0.05,{ }^{* *} p<0.01$, $* * * \mathrm{p}<0.001$. 

distinguish MCs from other INs. We analyzed unitary glutamatergic, excitatory and GABAergic, inhibitory currents (uEPSCs and uIPSCs, correspondingly) onto and from putative MCs in MC-PN connected pairs. One hallmark of MC connectivity is the strongly facilitating glutamatergic synaptic responses evoked upon PN action potentials (Wang et al., 2004; Kapfer et al., 2007; Silberberg and

Finally, we analyzed the kinetics of uIPSCs elicited by MCs and PV-cells in L2/3 PNs known to have distinctive kinetics (Silberberg et al., 2007). We found that uIPSCs evoked from MCs had significantly slower rise times as compared to PV INs (Rise time: $1.89 \pm 0.25 \mathrm{~ms}$ for GFP-expressing neurons and $0.57 \pm 0.02$ for PV INs; $p=2.2 e^{-5}$, unpaired T test; Fig. 1 I), consistent with characteristic MC-mediated dendritic uIPSCs.

Altogether, these results indicate that GFP-expressing neurons in L2/3 of the somatosensory cortex of X98 mice are a homogeneous subgroup of MCs as they exhibit typical anatomical, intrinsic excitability and synaptic features of MCs. Furthermore, this subgroup can be readily distinguished from the most abundant GABAergic PV INs.

\section{Martinotti Cells display target-specific synaptic properties}

In addition to PNs, SST INs were shown to contact other inhibitory neurons of the cortical microcircuits including VIP, PV and L1 INs (Pfeffer et al., 2013; Tremblay et al., 2016). However, it remains unknown whether this is true for MCs and whether these connections exhibit the biophysical and pharmacological properties observed in the inhibitory MC-PN synapse. In order to address this question, we systematically evoked uIPSCs from specific synapses formed between MCs and other INs using dual patch recordings in brain slices. To measure and compare evoked ulPSCs from pairs between MCs and other INs, we used brain slices containing differently labeled IN subtypes. For MC- 
PV synapses, we crossed X98 mice with Pvalb-tdTomato mice. For MC-L1 synapses, we used X98 mice and L1 INs were identified by their localization in L1. Finally, to record uIPSCs from MC-VIP cell pairs we crossed VIPcre with X98 mice. Mouse pups (P1-3) were then subjected to intracerebral injections of flexed AAV particles coding for tdTomato. We could thus obtain mice, in which MCs and VIP cells were simultaneously labeled with GFP and tdTomato, respectively. pairs), between MCs and L1 INs (11 connected out of 80 recorded pairs) and between MC and VIP INS (9 connected out of 45 recorded pairs; Fig. 2 B). Yet, the connectivity rate between MCs and these IN types was much lower than functional connections with PNs (30 connected out of 57 recorded pairs). Conversely, we did not find functional synaptic transmission between MCs (0 out of 10, connected/recorded pairs; Fig 2 B). Amplitudes of evoked uIPSCs were also largely variable between and within synapses. UIPSC amplitudes were consistently larger in MC-IN than in MC-PN synapses (uIPSC amplitudes: $10 \pm$ 1; $36 \pm 4 ; 72 \pm 32 ; 172 \pm 70$ pA; MC-PN, -PV, L1 and VIP INs, respectively; Kruskal Wallis followed by Mann-Withney with Bonferroni's correction; $n=10,7,5,6$, respectively). consequent electrotonic filtering. To further explore whether synaptic contacts made by MCs onto other circuit elements followed a similar pattern, we compared the kinetics of uIPSCs elicited by MCs onto PNs, PV-, L1-and VIP-INs (Fig. 2 C-F). Rise time (Rt) of MC-PN uIPSCs were significantly slower than those recorded from MC-PV, MC-L1 and MC-VIP-IN pairs $(1.89 \pm 0.25 ; 0.73 \pm 0.10 ; 0.63 \pm 0.13$; 
A

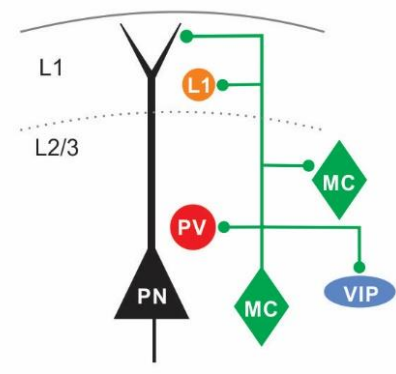

B

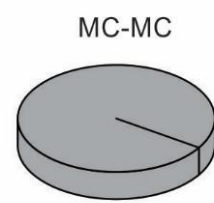

$n=10$
$0 \%$

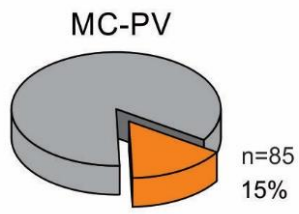

MC-L1IN

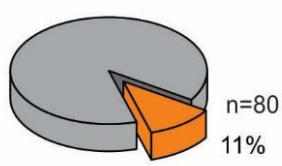

MC-PN

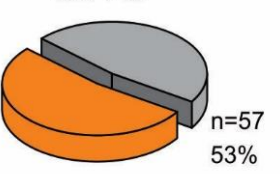

MC-VIP

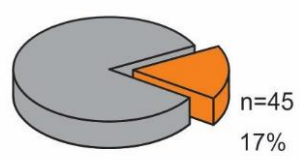

$17 \%$

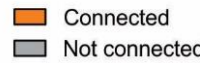

C

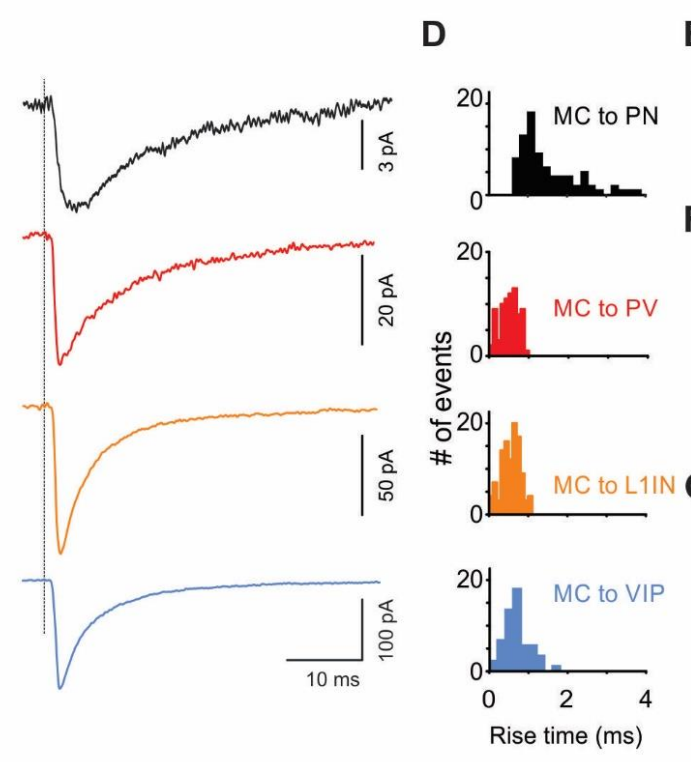

E
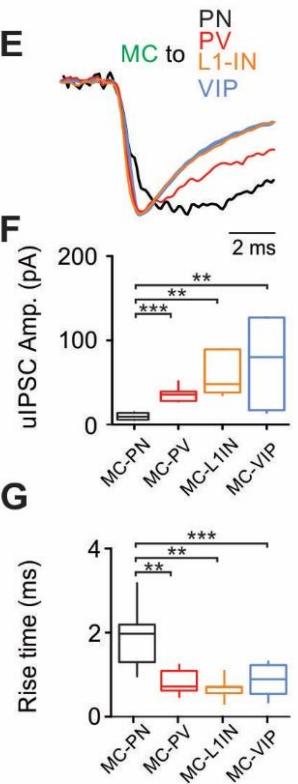

H

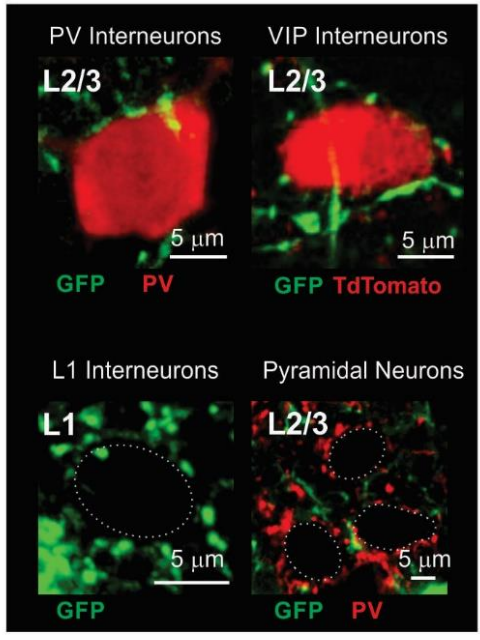

Figure 2: Diversity of MC synaptic contacts onto different neuronal types in the $L 2 / 3$ of somatosensory cortex.

A: Schematic representation of the tested inhibitory connections involving MCs. B: Pie charts illustrating the connectivity rates. C: Representative voltage-clamp uIPSCs average traces from MC-PN (black), MC-PV-IN (red) and MC-L1 IN (orange), VIP-INs (blue). Gray dotted line represents the time of the peak of presynaptic action potentials. D: Representative distributions of uIPSCs Rt recorded from individual MC-PN (top, black), MC-PVIN (middle, red) and MC-L1 (bottom, orange) connections. E: uIPSC (same as in C) normalized to the peak. F: Mean uIPSC amplitudes. G: Population plot of the mean uIPSCs Rts from individual MC-PN, MC-PV, MC-L1, PVPN and MC-VIP recorded connections. H: Confocal micrographs illustrating immunolabelling of: GFPexpressing MC puncta and PV (top left), GFP-expressing MC puncta and TdTomato-expressing VIP-IN (top right), GFP-expressing MC puncta around L1 IN soma (bottom left), GFP-expressing MC puncta and PV-positive synaptic baskets formed around L2/3 PN somas (bottom right). ${ }^{* *} \mathrm{p}<0.01,{ }^{* * *} \mathrm{p}<0.001$.

MCs could be that synaptic inputs located in the somatic/perisomatic region are less filtered than

to analyze putative contacts between GFP-positive MC axons and the somatic compartment of PV-, 
A

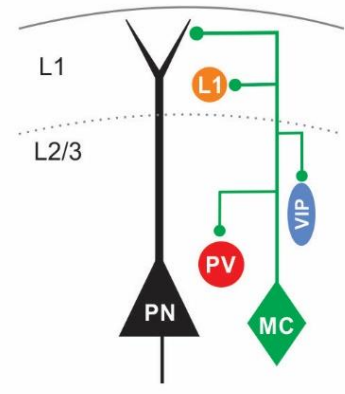

B

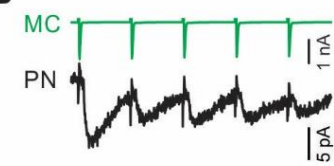

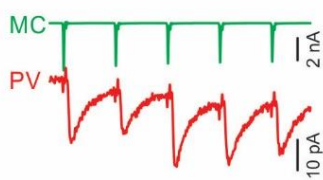
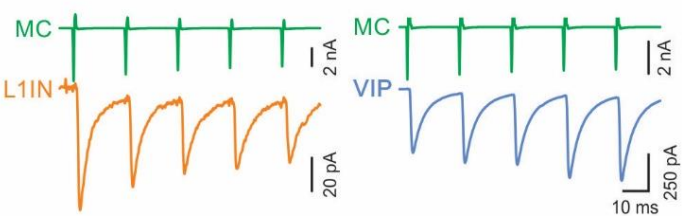

Figure 3: Plasticity of MC-mediated synaptic inhibition in L2/3 of somatosensory cortex.

A: Schematic representation of the tested inhibitory circuits involving MCs. B: Representative voltage-clamp averaged traces of uIPSCs from MCs onto PNs (black), PV-cells (red), L1-Ins (orange) and VIP-Ins (blue). C: Normalized uIPSC amplitude elicited by MCS onto different elements of the L2/3 inhibitory circuit. Inhibition of MCs onto PN (black) and L1-INs (orange) is strongly depressing whereas connections onto VIP (blue) are facilitating. A slight facilitation occurs at MC-PV synapses.

We then analyzed short-term synaptic plasticity (STP) at all unitary connections made by MCs with different postsynaptic targets (Fig. 3A), in response to trains of 5 action potentials at $50 \mathrm{~Hz}$. We found that short-term plasticity profiles depended on the postsynaptic target. Indeed, GABAergic transmission at MC-PN and MC-L1 IN synapses were strongly depressing. In contrast, MC-PV uIPSCs did not vary during the stimulus train, and MC-VIP synapse exhibited a significant facilitating profile (Fig. 3B-C). When compared with MC-PN connections, STP at MC-L1 IN synapses were not significantly different. However, STP of MC-PV and MC-VIP IN synapses was significantly different than MC-PN connections (repeated measures, two-way ANOVA followed by Bonferroni post hoc test; $F=24.1516$, 

negligible, extent PV, VIP and L1 INs, and avoid connecting between themselves. MC-dendrite targeting is specific for connections with PNs. Surprisingly, short-term plasticity at MC-synapses exhibit clear target specificity.

$\alpha 5-G A B A_{A} R s$ define MC-PN synapses in L2/3 of mouse somatosensory cortex

MC-PN synapse has been shown to be mediated by $\mathrm{GABA}_{\mathrm{A}} \mathrm{Rs}$ containing the $\alpha 5$ subunit in the rat somatosensory cortex (Ali and Thomson, 2008), in the mouse prefrontal cortex (Zorrilla de San Martin inhibition is sensitive to zolpidem (100 nM), a positive allosteric modulator, which at this concentration, is known to specifically bind the benzodiazepine site of $\alpha 1$-containing and, less efficiently, $\alpha 2$ - and $\alpha 3$-containing $\mathrm{GABA}_{A}$ receptors (Korpi et al., 2002; Möhler, 2002; Bacci et al., 2003). In order to validate these results in the mouse somatosensory cortex we tested the effects of $\alpha 5 \mathrm{IA}, \mathrm{a}$ negative allosteric modulator (NAM) specific for $\alpha 5-G A B A_{A}$ Rs (Dawson et al., 2006), and zolpidem on both MC-mediated PN dendritic inhibition and PV-IN-mediated PN perisomatic inhibition (Fig 4 A). PVPN uIPSC weighted decay time constant $\left(\tau_{d, w}\right)$ was significantly increased by zolpidem (control: $9.0 \pm$ $1.3 \mathrm{~ms}$; zolpidem: $11.2 \pm 0.7 \mathrm{~ms}, \mathrm{n}=6$ pairs, $\mathrm{p}=0.014$, Paired t-test; Fig 4 B). In contrast, PV-PN ulPSCs amplitude was unaffected by $\alpha 5 \mathrm{IA}$ (control: $63 \pm 22 \mathrm{pA}$; $\alpha 5 \mathrm{IA}: 65 \pm 20 \mathrm{pA}, \mathrm{n}=6$ pairs, $p=0.7294$, Paired t-test; Fig. 4 B). The amplitude of uIPSCs elicited from MCs were highly sensitive to $\alpha 5 \mathrm{~A}$ (control: 177 $\pm 44 \mathrm{pA} ; \alpha 5 \mathrm{IA}: 104 \pm 23 \mathrm{pA}, \mathrm{n}=11$ pairs; $\mathrm{p}=0.003$, Wilcoxon Signed-Ranks test; Fig 4 C), and zolpidem did not affect the weighted decay time constant of the MC-PN uIPSCs (control: $8.2 \pm 1.2$ ms; zolpidem: efficacy, thus not providing a complete blockade of $\alpha 5-G A B A_{A} R s$ (Dawson et al., 2006). Importantly, 

dendritic inhibition at specific synapses made by MCs in the cortex and by OLM interneurons in the hippocampus (Ali and Thomson, 2008; Schulz et al., 2018; Zorrilla de San Martin et al., 2020). It is possible that sensitivity of uIPSCs to $\alpha 5$ IA could be partially or fully due to activation of extrasynaptic $\mathrm{GABA}_{\mathrm{A}}$ Rs due to GABA spillover, induced by AP-evoked synaptic transmission. To further study the role of synaptic $\alpha 5-G A B A_{A} R s$, we measured spontaneous inhibitory postsynaptic currents (sIPSCs) recorded from PNs (Fig 4 D-F). Because quantal, AP-independent synaptic events make up a large fraction of sIPSCs, these are less likely shaped by activation of extrasynaptic receptors. To separate putative dendritic and perisomatic events, we sorted sIPSCs into two groups based on their rise-times (Fig 4 D-F). We considered the events with rise times larger than $1.8 \mathrm{~ms}$ as 'slow', whereas those with rise-times smaller than $1.8 \mathrm{~ms}$ were defined as 'fast', based on the average rise-time obtained at connected MC-PN pairs (Figs. 1I; 2C-E). The amplitudes of slow sIPSCs were significantly reduced after 10 minutes incubation with $100 \mathrm{nM} \alpha 5 \mathrm{IA}$ (control: $31 \pm 2 \mathrm{pA}, \alpha 5 \mathrm{IA}: 28 \pm 1 \mathrm{pA}, \mathrm{n}=11$ cells, $p=0.03$, Wilcoxon Signed-Ranks test; Fig. 4 E). Conversely, the same concentration of $\alpha 5$ IA did not affect fast sIPSCs amplitude (control: $38 \pm 2$ pA, $\alpha 5 I A: 36 \pm 2 p A ; n=11$ cells, $p=0.3636$, Wilcoxon Signed-Ranks types, not using $\alpha 5-G A B A_{A} R s$. 
A
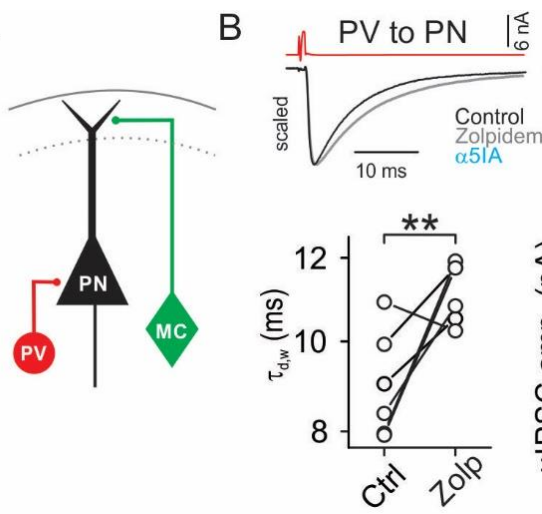
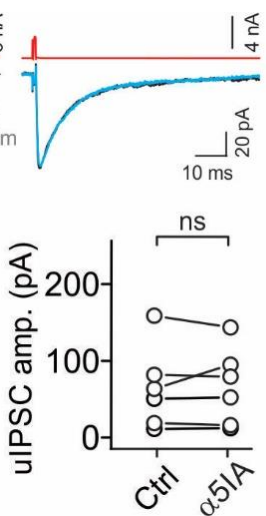
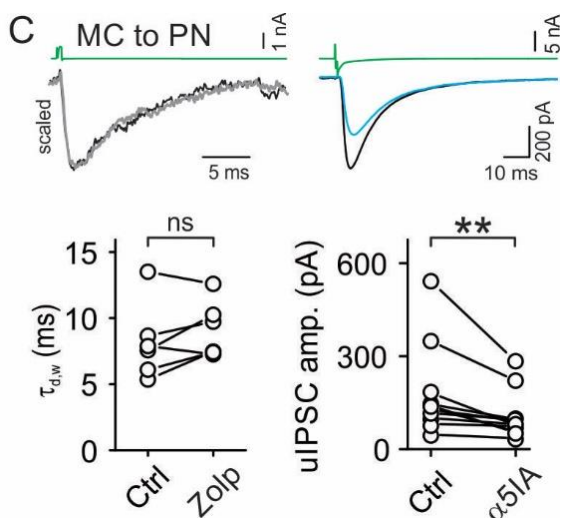

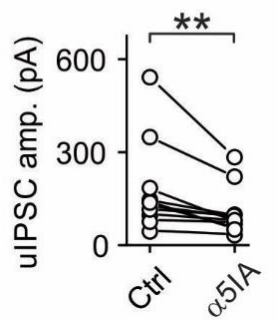

D

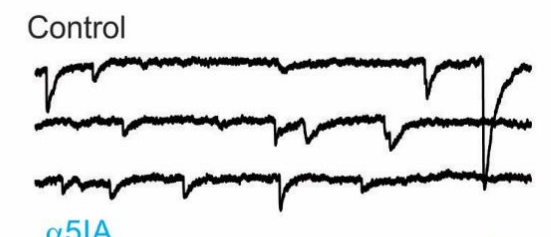

$E$

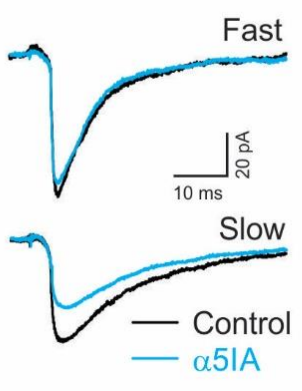

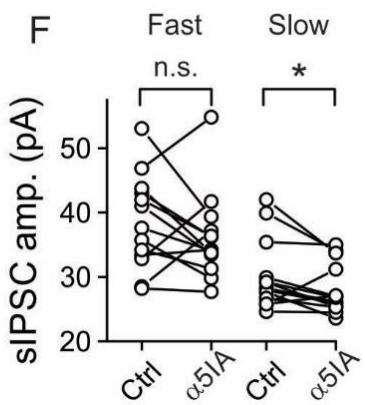

Figure 4: $\alpha 5-G A B A_{A} R s$ mediate synaptic inhibition selectively from MCs.

A: Schematic of paired recordings between a MC or PV-IN and a PN. B: Top left, Representative average uIPSC elicited by a PV-INs onto a PN in the absence (black) and presence (grey) of zolpidem. Traces are scaled to highlight zolpidem effect on uIPSC decay time. Bottom left, population data of zolpidem effect on the weighted decay time constant ( $\tau_{d, w}$, left) and $\alpha 5$ IA effect on uIPSC amplitude in PV-PN pairs. Top right: representative average uIPSC traces elicited a PV cell onto a PN in the absence (black) and presence (blue) of $\alpha 5$ IA. C: Same as in B for MC-PN pairs. D: Representative voltage-clamp traces of sIPSCs recorded from a PN before (control, black) and after 15 minutes incubation with $100 \mathrm{nM} \alpha 5 \mathrm{IA}$ (blue). E: Representative averaged traces of fast (top) and slow (bottom) events recorded from MCs. Only amplitudes of slow events are affected by $\alpha 5 I A$ (blue trace, bottom panel). F: Population plot of individual cells, fast and slow sIPSCs median amplitudes measured in control and after incubation with $\alpha 5$ IA. ${ }^{*} p<0.05,{ }^{*} p<0.01$. 
MCs inhibit PNs, but not other interneurons, through $\alpha 5-G A B A_{A} R s$.

408

409

410

411

412

413

414

In the previous sections, we showed that MCs make synaptic contacts exhibiting target-specific biophysical and physiological properties. We also showed that, among the inhibitory inputs received by PNs, those originating from MCs are distinguished by their sensitivity to $\alpha 5 \mathrm{IA}$. We therefore tested whether postsynaptic expression of $\alpha 5-G A B A_{A} R s$ is a trait shared by all synaptic contacts made by MCs or it is specific for synaptic contacts that MCs form on PN dendrites. To address this question, we measured unitary GABAergic synaptic transmission between MCs and other INs and tested their sensitivity to $\alpha 5$ IA. We found that uIPSC amplitudes elicited by MCs and recorded in PV INs (control: $31 \pm 4 \mathrm{pA}, \alpha 5 \mathrm{~A}: 35 \pm 6 \mathrm{pA}, \mathrm{n}=11$ pairs, $p=0.3757$, paired t test) , L1 INs (control: $83 \pm 32 \mathrm{pA}, \alpha 5 \mathrm{~A}: 102$ $\pm 29 \mathrm{pA}, \mathrm{n}=5$ pairs, $p=0.3757$, paired t test) and VIP INs (control: $178 \pm 100 \mathrm{pA}, \alpha 5 \mathrm{~A}: 118 \pm 52 \mathrm{pA}, \mathrm{n}=4$ pairs, $p=0.7432$, Wilcoxon signed ranks test) were not sensitive to $\alpha 5 \mathrm{IA}$ (Fig. $5 \mathrm{~B}-\mathrm{C}$ ).

In the hippocampus, SST-positive, OL-M INs receive $\alpha 5$-mediated inhibition from VIP INs (Magnin et al., 2019). Since VIP-MC synapses represent an important disinhibitory circuit in the cortex as well, we asked whether $\alpha 5-G_{A B A}$ Rs mediate inhibitory inputs from VIP INs also in the mouse somatosensory cortex. To address this question and to activate VIP INs specifically, while recording from GFP-expressing MCs, we crossed VIP-cre mice with X98 mice. Since dual whole-cell recordings showed a very low yield ( 3 connected out of 45 recorded pairs), we expressed the light-sensitive opsin ChR2 via injection of flexed-ChR2 AAV particles in the barrel cortex of VIPCre::X98 1-3-days-old pups. We recorded light-evoked IPSCs in MCs, and found that inhibitory responses originating at VIP cells were not sensitive to $\alpha 5 \mathrm{IA}$ (control: $170 \pm 46 \mathrm{pA}, \alpha 5 \mathrm{IA}: 166 \pm 52 \mathrm{pA}, \mathrm{n}=7, \mathrm{p}=0.7432$, Wilcoxon signed ranks test; Fig 5 B). Furthermore, the amplitude of sIPSCs recorded from MCs were not affected by incubation with $100 \mathrm{nM} \alpha 5 \mathrm{IA}$ (control: $32 \pm 1 \mathrm{pA}, \alpha 5 \mathrm{IA}: 29 \pm 3 \mathrm{pA}, \mathrm{n}=26, \mathrm{p}=0.0659$, Wilcoxon signed ranks test; Fig $5 \mathrm{E})$.

Overall, these results indicate that GABAergic inhibition to and from MCs uses $\alpha 5-G A B A_{A} R s$ exclusively at synapses formed with PN distal dendrites and not for other MC-targets within cortical 


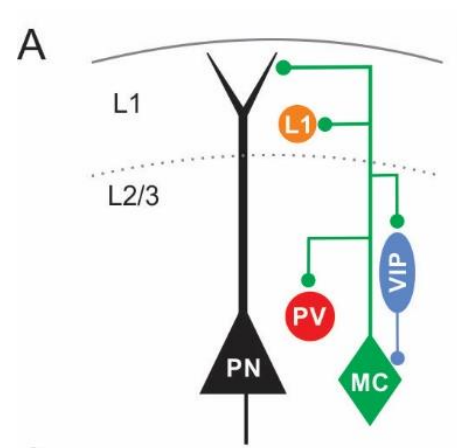

C MC to PV

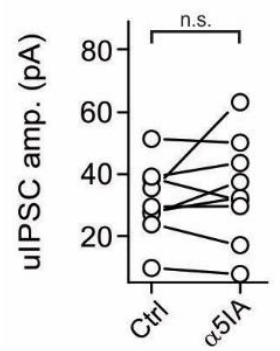

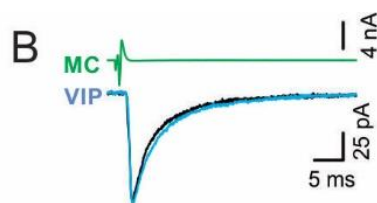
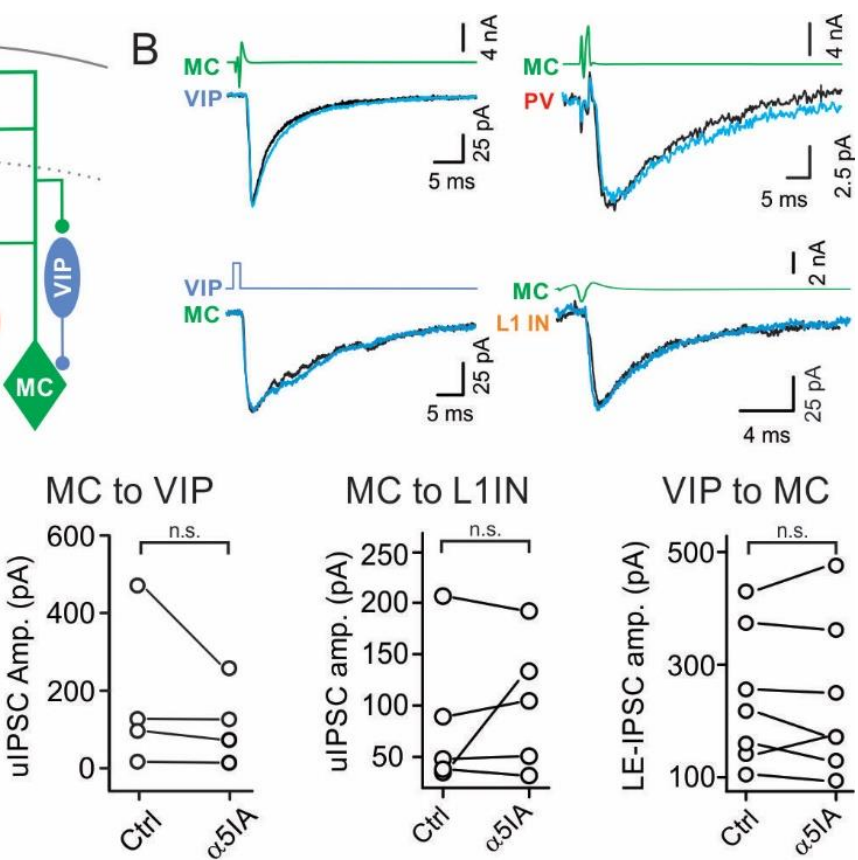

VIP to MC

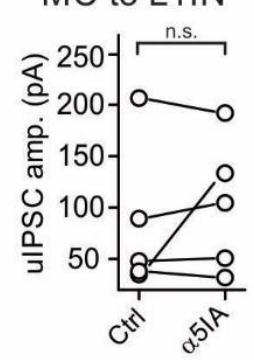

Figure 5: Inhibitory synaptic transmission involving MCs and other interneurons does not rely on $\alpha 5$ GABA $_{A}$ Rs.

A: Schematic representation of the tested inhibitory circuits involving MCs. B: Representative averaged voltage-clamp traces of uIPSCs from MCs onto different element of the circuit and from VIP to MC before (black) and after (blue) application of a5IA. C: Population data of uIPSC amplitude before (Ctrl) and 15 minutes after $\alpha 5 \mathrm{IA}$ application.

Tonic inhibition is mediated by $\alpha 5-\mathrm{GABA}_{\mathrm{A}} \mathrm{Rs}$ in PN, but not MC nor PV-IN.

$\alpha 5-G A B A_{A} R s$ have been largely associated to tonic inhibition due to extrasynaptic immunoreactivity

in cell culture (Loebrich et al., 2006; Serwanski et al., 2006), hippocampus and cortex (Serwanski et al., 2006) and amygdala (Botta et al., 2015) and the lack of tonic inhibitory current in hippocampal PNs of a5 knock-out mice (Caraiscos et al., 2004). then quantified the difference in holding current amplitude $\left(\Delta \mathrm{I}_{\text {hold }}\right)$ before and after bath application of $1 \mu \mathrm{M}$ gabazine (Fig. 6). Pre-incubation with $100 \mathrm{nM} \alpha 5 \mathrm{IA}$ significantly reduced GABAergic $\Delta \mathrm{l}_{\text {hold }}$ in 
$444 p=0.0244$, unpaired t test; Fig. 6 A,B). A similar percentage of reduction was obtained incubating with

$445500 \mathrm{nM} \alpha 5 \mathrm{IA}$ (data not shown), ruling out that $\alpha 5-\mathrm{GABA}_{\mathrm{A}} \mathrm{Rs}$ required higher drug concentrations.

446 Conversely, incubation with $\alpha 5$ IA failed to produce any significant change in tonic current recorded

447 from MCs (ACSF: $16 \pm 5$ pA, n=9; $\alpha 5 \mid A: 23 \pm 6$ pA, $n=14, p=0.3330$, unpaired t test; Fig. 6 C,D) nor PV

448 INs (ACSF: $33 \pm 10$ pA, $n=9 ; \alpha 5 I A: 24 \pm 5$ pA, $n=14, p=0.6591$, unpaired t test; Fig. $6 E, F$ ), showing that

449 tonic inhibition is mediated by $\alpha 5-G A B A_{A} R s$ exclusively in PNs.

450 Altogether, these results indicate that $\alpha 5-G A B A_{A} R s$ are selectively expressed by PNs and they

451 mediate both tonic and dendritic, phasic synaptic inhibition. 
A

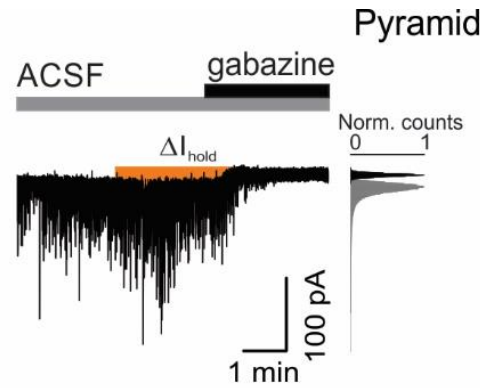

C

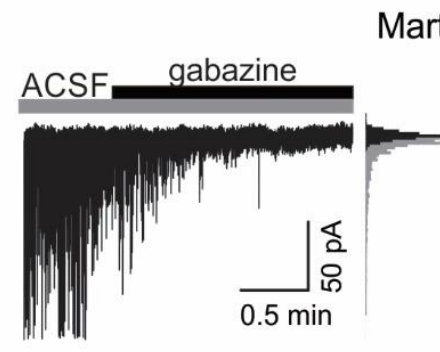

Martinotti Cells
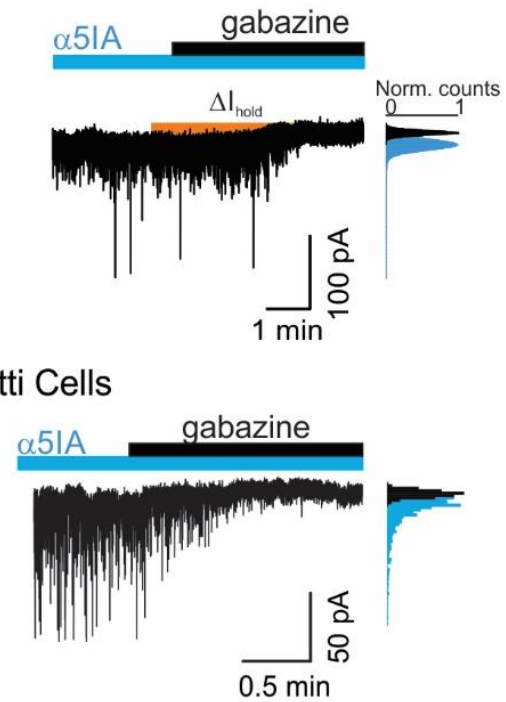

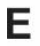

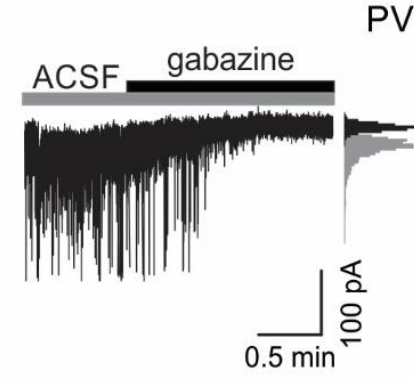

$\mathrm{PV}$ interneurons

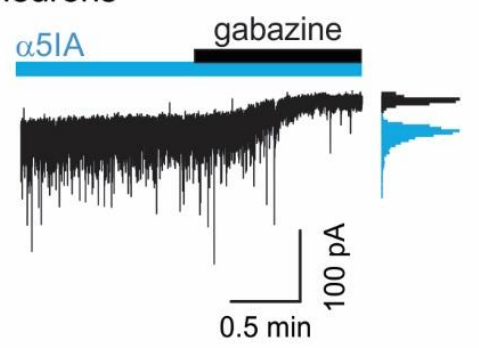

B
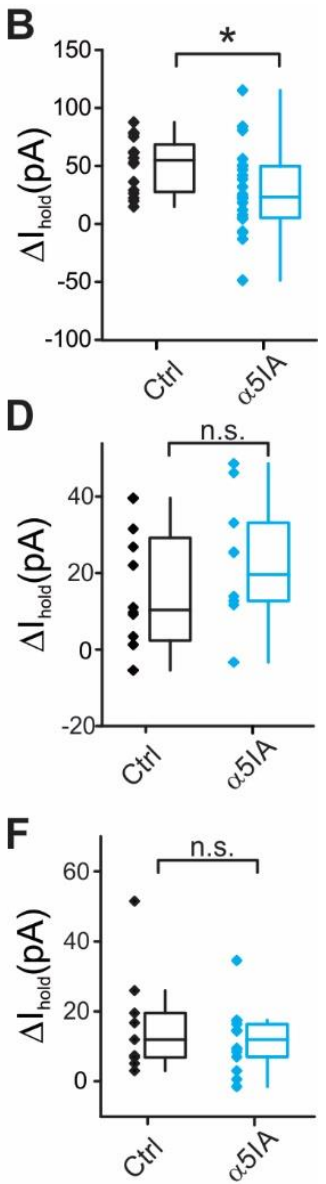

Figure 6: $\alpha 5 \mathrm{GABA}_{\mathrm{A}} \mathrm{Rs}$ only contribute to tonic inhibition in L2/3 PN of mouse somatosensory cortex.

A: Whole-cell voltage-clamp recordings from L2/3 PNs preincubated with either vehicle (aCSF, left) or $\alpha 5 I A$ (right). DNQX $(10 \mu \mathrm{M})$ and GABA $(5 \mu \mathrm{M})$ were continuously present in both conditions. Orange areas ( $\Delta$ lhold) represent tonic inhibition measured after gabazine onset (dotted red line). Insets: All-points histograms of the current trace obtained in the absence (grey and blue histograms) and presence of gabazine (black histograms). Gaussian fits were used to determine the noise half-width. C-E: same as in A for MCs and PVINs, correspondingly. B-D-F: Population graphs of holding-current shifts after gabazine application $\left(\Delta \mathrm{l}_{\text {hold }}\right) .{ }^{*}$ $p<0.05$.

Discussion

In this study, we explored physiological aspects of different inhibitory synaptic connections made

by MCs in the L2/3 of the mouse somatosensory cortex. We show that inhibitory synapses made by 
459 VIP and L1 inhibitory INs also receive significant inhibition from MCs. Notably, we showed that the 460 MC-PN synapse is distinguished by two unique features: slow kinetics and expression of the $\alpha 5-$ $G A B A_{A} R$. Finally, we showed that $\alpha 5-G A B A_{A} R s$ contribute to tonic inhibition of PNs but not of other INs, confirming the specific involvement of this receptor in PN dendritic inhibition. manipulating and recording their activity, using cre-driven expression of light-sensitive opsins or genetically encoded $\mathrm{Ca}^{2+}$ sensors (Taniguchi et al., 2011). Despite its extensive use, the SST-cre mouse connectivity (Halabisky et al., 2006; Ma et al., 2006; McGarry et al., 2010; Naka et al., 2019). Therefore, it is important to focus the study of SST-positive neurons to defined homogeneous subpopulations, in order to prevent unwanted over-simplified conclusions. MCs represent a specific subpopulation of GABAergic interneurons, accounting for only $20 \%$ of all SST-expressing cells (Yavorska and Wehr, 2016). Here we used the $X 98$ mouse line to study MCs specifically (Ma et al., 2006). We provide evidence that GFP-expressing neurons in the somatosensory cortex from the X98 mouse line exhibit the typical anatomical and electrophysiological properties of MCs (Wang et al., 2004; Kapfer et al., 2007; Silberberg and Markram, 2007; Tremblay et al., 2016). In addition, glutamatergic recruitment of GFP-positive cells is strongly facilitating, as opposed to PV cells, another hallmark of MCs (Reyes et al., 1998). Therefore, we conclude that this mouse line represents an excellent tool to study inhibitory circuits involving MCs. cortical microcircuits, and, in addition, they are targeted by VIP-expressing interneurons (Pfeffer et al., 2013; Kepecs and Fishell, 2014; Tremblay et al., 2016; Walker et al., 2016). We found that MCs contact PV- VIP- and L1-INs at a reduced connectivity rate, as compared to MC-PN connections. Moreover, use $\alpha 5-G A B A_{A} R s$. 
MCs were hypothesized to provide a non-specific 'blanket' of inhibition to PNs (Fino and Yuste, 2011; Fino et al., 2013; Karnani et al., 2016). Accordingly, we found a relatively high connectivity rates between MCs and L2/3 PNs, consistent with the prominent MC axonal plexus innervating L1. However, our results indicate that despite extensively innervating $L 1, M C$ axons possess a very strong tropism for PN dendrites. Yet, despite at lower rate, MCs do contact also L1-INs, which exert slow feed-forward between $\mathrm{MC}$ axons on the perisomatic region of PNs. Conversely, we found $\mathrm{MC}$ putative boutons juxtaposed to the perisomatic region of PV, VIP and L1 INs. This is consistent with the fast, non-filtered, IPSCs observed in somatic whole-cell recordings and in line with a previous report showing that inhibitory contacts onto PV INs are preferentially located in the proximal dendrites and soma while excitatory inputs are located in distal dendrites (Kameda et al., 2012). biophysical presynaptic properties, such as low or high release probability, respectively (Jackman and originating from the same cell type, was described at glutamatergic synapses from PNs recruiting different IN subtypes in the neocortex and hippocampus (Reyes et al., 1998). Our finding indicates that single-axon, target-specific bidirectional short-term plasticity occurs also at GABAergic synapses. Intriguingly, synapses made in L1 (with either PN distal dendrites or sparse INs) are depressing, 
whereas, inhibitory connection that the same cells make onto their targets in L2/3 (PV and VIP cells) are either uniform or strongly facilitating. It will be interesting to determine the molecular and synaptic mechanisms by which the identity of the postsynaptic neuron determines the efficacy of GABAergic synapses originating from the same MC. Target cell type-dependent variability in presynaptic properties increases the computational power of neuronal networks. It will be therefore synaptic efficacy. modulate input onto PNs and they likely control, at least in part, the output activity of other interneurons. Therefore, inhibitory circuits formed by MCs seem to exhibit a more complex architecture and function than previously hypothesized as provider of a mere blanket of inhibition (Fino and Yuste, 2011).

In addition to the strong preference for distal apical dendrites, MCs display another PN-specific synaptic feature, as they use $\alpha 5-G A B A_{A} R s$ for synaptic dendritic inhibition. Indeed, GABAergic synapses from MCs to other interneurons are perisomatic and do not use $\alpha 5-G A B A_{A} R s$. In fact, lack of $\alpha 5 \mathrm{IA}$ effects on tonic inhibition on PV and MCs suggest that these major IN subtypes do not express this $\mathrm{GABA}_{A} \mathrm{R} \alpha$ subunit. Interestingly, It has been recently reported that hippocampal OriensLacunosum Moleculare INs also express, $\alpha 5-G A B A_{A} R s$ at synapses originating at VIP INs (Magnin et al., 2019). Yet, we did not find evidence of $\alpha 5 I A$ effect on VIP-IN-evoked IPSCs in MCs of the barrel cortex, suggesting that cortical MCs differ from their hippocampal counterparts. The $\alpha 5$ subunit is much more strongly expressed in the hippocampus than in the neocortex (Lingford-Hughes et al., 2002). Therefore, it will be interesting to reveal whether $\alpha 5$ has different circuit-specificity and/or plays a different role in these two areas. Likewise, it remains to be tested whether $\alpha 5-G A B A_{A} R s$ are also expressed by other subtypes of inhibitory neurons. Our results on L1-INs suggest that MCs do not use $\alpha 5-G A B A_{A} R s$ at these synapses. However, L1 is populated by a heterogeneous IN population (Schuman 
et al., 2019) and, since we did not use specific mouse lines to target distinct cell types, our data may have been collected from a relatively heterogeneous interneuron group.

In addition to dendritic filtering, MC-PN synaptic responses might be slow due to the specific properties of the $\alpha 5$-subunit itself, which is exclusively expressed at this synapse. The slow kinetics and the rectification of $\alpha 5-G A B A_{A}$ Rs match the biophysical properties of NMDARs, which govern $\mathrm{Ca}^{2+}$ signaling and dendritic computation in PNs (Branco and Häusser, 2010; Tran-Van-Minh et al., 2015; series resistance typical of dendritic patch recordings might prevent an accurate analysis of fast currents. concentrations of GABA, their non-desensitizing properties and the lack of evidence supporting its implication in synaptic transmission (Caraiscos et al., 2004). However, knock down of radixin, the extrasynaptic scaffolding protein associated to $\alpha 5-G A B A_{A} R s$ did not produce any effect on GABA evoked current, suggesting that extrasynaptic $\alpha 5-\mathrm{GABA}_{\mathrm{A}} \mathrm{Rs}$ might not be functional (Loebrich et al., 2006). Furthermore, the participation of $\alpha 5-G A B A_{A} R s$ in phasic synaptic inhibition has been recently demonstrated in different brain structures, namely the rat somatosensory cortex (Ali and Thomson, 2008), mouse hippocampus (Schulz et al., 2018) and mouse prefrontal cortex (Zorrilla de San Martin et al., 2020). Even for action potential-dependent unitary responses between MCs and PNs, it is possible that GABA could spill over to peri- or extrasynaptic $G A B A_{A} R s$ containing $\alpha 5$. If this were the case, we would not have detected significant effects on quantal events, which reflect mostly purely synaptic activation of GABA $A_{A} R s$. Importantly, we recorded sIPSCs from the soma of $L 2 / 3$ PNs and found that only slow sIPSCs were sensitive to $\alpha 5 \mathrm{IA}$, whereas fast perisomatic inhibitory events were unaffected. Our results on sIPSCs corroborate the synaptic localization of $\alpha 5-G A B A_{A} R s$. Indeed, at our extracellular $\mathrm{K}^{+}$concentrations, sIPSCS are dominated by AP independent miniature events. The blockade of MC-PN uIPSCs, slow sIPSCs and tonic inhibition was not total but it was in all cases maximal, taking into account the actual efficacy ( 40\%) of $\alpha 5$ IA (Sternfeld et al., 2004; Atack, 2010). 

$\mathrm{GABA}_{A} \mathrm{Rs}$ are prominently expressed at synaptic sites of dendritic MC-PN connections and are responsible for dendritic inhibition from this specific GABAergic neuron type. In fact, the $\alpha 5$-mediated tonic currents could be the direct activation by ambient GABA of high affinity synaptic, and not necessarily extrasynaptic receptors. addition to the high $\alpha 5-G A B A_{A} R s$ expression in the mouse prefrontal cortex and hippocampus (Fritschy and Mohler, 1995) led to propose novel potential pro-cognitive pharmacological strategies. This strategy is being actively explored to treat intellectual disability in Down syndrome (Braudeau et al., 2011; Martínez-Cué et al., 2013; Duchon et al., 2019; Zorrilla de San Martin et al., 2020) and in other brain diseases characterized by memory impairments (Zurek et al., 2014) and depressive states (Zanos et al., 2017). Specific negative modulation of these receptors would facilitate cognition avoiding anxiogenic and pro-convulsive effects of wide spectrum $G_{A B A} R s$ antagonists due to the restricted expression of the $\alpha 5$ subunit to this specific inhibitory circuit formed by MCs.

\section{References}

Abs E, Poorthuis RB, Apelblat D, Muhammad K, Pardi MB, Enke L, Kushinsky D, Pu D-L, Eizinger MF, Layer 1 Interneurons. Neuron:1-16. in visual cortex. Nature 490:226-230. 
Ali AB, Thomson AM (2008) Synaptic $\alpha 5$ subunit-containing GABAA receptors mediate ipsps elicited by dendrite-preferring cells in rat neocortex. Cereb Cortex 18:1260-1271.

Atack JR (2010) Preclinical and clinical pharmacology of the GABAA receptor $\alpha 5$ subtype-selective inverse agonist $\alpha 5$ IA. Pharmacol Ther 125:11-26.

Bacci A, Rudolph U, Huguenard JR, Prince DA (2003) Major Differences in Inhibitory Synaptic Transmission onto Two Neocortical Interneuron Subclasses. J Neurosci 23:9664-9674.

Bartos M, Vida I, Jonas P (2007) Synaptic mechanisms of synchronized gamma oscillations in inhibitory interneuron networks. Nat Rev Neurosci 8:45-56.

Berger TK, Perin R, Silberberg G, Markram H, Berger TK (2009) Frequency-dependent disynaptic inhibition in the pyramidal network: a ubiquitous pathway in the developing rat neocortex. J Physiol 58722:5411-5425.

Botta P, Demmou L, Kasugai Y, Markovic M, Xu C, Fadok JP, Lu T, Poe MM, Xu L, Cook JM, Rudolph U, Sah P, Ferraguti F, Lüthi A (2015) Regulating anxiety with extrasynaptic inhibition. Nat Neurosci 18:1493-1500.

Branco T, Häusser M (2010) The single dendritic branch as a fundamental functional unit in the nervous system. Curr Opin Neurobiol 20:494-502.

Braudeau J, Delatour B, Duchon A, Pereira PL, Dauphinot L, de Chaumont F, Olivo-Marin J-C, Dodd R, Hérault Y, Potier M-C (2011) Specific targeting of the GABA-A receptor \{alpha\}5 subtype by a selective inverse agonist restores cognitive deficits in Down syndrome mice. J Psychopharmacol 25:1030-1042.

Buzsáki G (2010) Neural Syntax: Cell Assemblies, Synapsembles, and Readers. Neuron 68:362-385.

Buzsáki G, Wang X-J (2012) Mechanisms of Gamma Oscillations. Annu Rev Neurosci 35:203-225.

Caraiscos VB, Elliott EM, You-Ten KE, Cheng VY, Belelli D, Newell JG, Jackson MF, Lambert JJ, Rosahl TW, Wafford K a, MacDonald JF, Orser B a (2004) Tonic inhibition in mouse hippocampal CA1 

pyramidal neurons is mediated by alpha5 subunit-containing gamma-aminobutyric acid type $\mathrm{A}$ receptors. Proc Natl Acad Sci U S A 101:3662-3667.

Clem RL, Cummings KA (2020) Prefrontal somatostatin interneurons encode fear memory. Nat Neurosci 23:p61, 14 p.

Collinson N, Kuenzi FM, Jarolimek W, Maubach K a, Cothliff R, Sur C, Smith A, Otu FM, Howell O, Atack JR, McKernan RM, Seabrook GR, Dawson GR, Whiting PJ, Rosahl TW (2002) Enhanced learning and memory and altered GABAergic synaptic transmission in mice lacking the alpha 5 subunit of the GABAA receptor. J Neurosci 22:5572-5580.

Dawson GR, Maubach KA, Collinson N, Cobain M, Everitt BJ, MacLeod AM, Choudhury HI, McDonald LM, Pillai G, Rycroft W, Smith AJ, Sternfeld F, Tattersall FD, Wafford KA, Reynolds DS, Seabrook GR, Atack JR (2006) An inverse agonist selective for $\alpha 5$ subunit-containing GABA A receptors MC (2019) Long-lasting correction of in vivo LTP and cognitive deficits of mice modelling Down

Fino E, Packer AM, Yuste R (2013) The logic of inhibitory connectivity in the neocortex. Neuroscientist syndrome with an $\alpha 5$-selective GABAA inverse agonist. Br J Pharmacol 177:1106-1118.

Duncan CE, Webster MJ, Rothmond DA, Bahn S, Elashoff M, Shannon Weickert C (2010) Prefrontal GABAA receptor $\alpha$-subunit expression in normal postnatal human development and schizophrenia. J Psychiatr Res 44:673-681.

Fino E, Yuste R (2011) Dense inhibitory connectivity in neocortex. Neuron 69:1188-1203.

Fritschy JM, Mohler H (1995) GABAA receptor heterogeneity in the adult rat brain: Differential regional and cellular distribution of seven major subunits. J Comp Neurol 359:154-194. 
in Schizophrenia. Curr Pharm Des 20:5069-5076.

Gouwens NW et al. (2020) Integrated Morphoelectric and Transcriptomic Classification of Cortical GABAergic Cells. Cell 183:935-953.e19.

Halabisky B, Shen F, Huguenard JR, Prince DA (2006) Electrophysiological classification of somatostatin-positive interneurons in mouse sensorimotor cortex. J Neurophysiol 96:834-845.

Hilscher MM, Leão RN, Edwards SJ, Leão KE, Kullander K, Bacci A (2016) Chrna2-Martinotti Cells Synchronize layer 5 type A Pyramidal Cells via Rebound Excitation. PLoS Biol 15.

Isaacson JS, Scanziani M (2011) How inhibition shapes cortical activity. Neuron 72:231-243.

Jackman SL, Regehr WG (2017) The Mechanisms and Functions of Synaptic Facilitation. Neuron 94:447-464.

Kameda H, Hioki H, Tanaka YH, Tanaka T, Sohn J, Sonomura T, Furuta T, Fujiyama F, Kaneko T (2012) Parvalbumin-producing cortical interneurons receive inhibitory inputs on proximal portions and cortical excitatory inputs on distal dendrites. Eur J Neurosci 35:838-854.

Kapfer C, Glickfeld LL, Atallah B V, Scanziani M (2007) Supralinear increase of recurrent inhibition during sparse activity in the somatosensory cortex. Nat Neurosci 10:743-753.

Karnani MM, Jackson J, Ayzenshtat I, Sichani XH, Manoocheri K, Kim S, Yuste R (2016) Opening holes in the blanket of inhibition: Localized lateral disinhibition by vip interneurons. J Neurosci $36: 3471-3480$.

Kepecs A, Fishell G (2014) Interneuron cell types are fit to function. Nature 505:318-326.

Korpi ER, Mihalek RM, Sinkkonen ST, Hauer B, Hevers W, Homanics GE, Sieghart W, Lüddens H (2002) Altered receptor subtypes in the forebrain of GABAA receptor $\delta$ subunit-deficient mice: Recruitment of $\gamma 2$ subunits. Neuroscience 109:733-743.

Letzkus JJ, Wolff SBE, Meyer EMM, Tovote P, Courtin J, Herry C, Lüthi A (2011) A disinhibitory microcircuit for associative fear learning in the auditory cortex. Nature 480:331-335. 
Lingford-Hughes A, Hume SP, Feeney A, Hirani E, Osman S, Cunningham VJ, Pike VW, Brooks DJ, Nutt DJ (2002) Imaging the GABA-benzodiazepine receptor subtype containing the alpha5-subunit in vivo with [11C]Ro15 4513 positron emission tomography. J Cereb Blood Flow Metab 22:878889.

Loebrich S, Bä Hring R, Katsuno T, Tsukita S, Kneussel M (2006) Activated radixin is essential for GABA A receptor a5 subunit anchoring at the actin cytoskeleton. EMBO J 25:987-999.

Lovett-Barron M, Turi GF, Kaifosh P, Lee PH, Bolze F, Sun X-HH, Nicoud J-FF, Zemelman B V, Sternson SM, Losonczy A (2012) Regulation of neuronal input transformations by tunable dendritic inhibition. Nat Neurosci 15:423-430.

Ma Y, Hu H, Berrebi AS, Mathers PH, Agmon A (2006) Distinct subtypes of somatostatin-containing neocortical interneurons revealed in transgenic mice. J Neurosci 26:5069-5082.

Magnin E, Francavilla R, Amalyan S, Gervais E, David LS, Luo X, Topolnik L (2019) Input-specific synaptic location and function of the $\alpha 5$ GABA a receptor subunit in the mouse CA1 hippocampal neurons. J Neurosci 39:788-801.

Martínez-Cué C, Martinez P, Rueda N, Vidal R, Garcia S, Vidal V, Corrales A, Montero JA, Pazos A, Florez J, Gasser R, Thomas AW, Honer M, Knoflach F, Trejo JL, Wettstein JG, Hernandez M-C (2013) Reducing GABAA 5 Receptor-Mediated Inhibition Rescues Functional and Neuromorphological Deficits in a Mouse Model of Down Syndrome. J Neurosci 33:3953-3966.

McGarry LM, Packer AM, Fino E, Nikolenko V, Sippy T, Yuste R (2010) Quantitative classifi cation of somatostatin-positive neocortical interneurons identifi es three interneuron subtypes. Front Neural Circuits 4.

Möhler H (2002) Pathophysiological aspects of diversity in neuronal inhibition: A new benzodiazepine pharmacology. Dialogues Clin Neurosci 4:261-269.

Naka A, Veit J, Shababo B, Chance RK, Risso D, Stafford D, Snyder B, Egladyous A, Chu D, Sridharan S, 

somatostatin interneurons enforce layer specific control. Elife 8:1-36.

685

686

687

688

689

690

691

692

693

694

695

696

697

698

699

700

701

702

Paul A, Crow M, Raudales R, He M, Gillis J, Huang ZJ (2017) Transcriptional Architecture of Synaptic Communication Delineates GABAergic Neuron Identity. Cell 171:522-539.e20.

Pfeffer CK, Xue M, He M, Huang ZJ, Scanziani M (2013) Inhibition of inhibition in visual cortex: The logic of connections between molecularly distinct interneurons. Nat Neurosci 16:1068-1076.

Reyes A, Lujan R, Rozov A, Burnashev N, Somogyi P, Sakmann B (1998) Target-cell-specific facilitation and depression in neocortical circuits. Nat Neurosci 1:279-285.

Rusakov DA, Fine A (2003) Extracellular Ca2+ depletion contributes to fast activity-dependent modulation of synaptic transmission in the brain. Neuron 37:287-297.

Scala F, Kobak D, Shan S, Bernaerts Y, Laturnus S, Cadwell CR, Hartmanis L, Froudarakis E, Castro JR, Tan ZH, Papadopoulos S, Patel SS, Sandberg R, Berens P, Jiang X, Tolias AS (2019) Layer 4 of mouse neocortex differs in cell types and circuit organization between sensory areas. Nat Commun 10.

Scheggia D, Manago F, Maltese F, Bruni S, Nigro M, Latuske P, Contarini G, Gomez-Gonzola M, Requie LM, Ferretti V, Castellani G, Mauro D, Bonavia A, Carmignoto G, Yizhar O, Papaleo F (2019) Somatostatin interneurons in the prefrontal cortex control affective state discrimination in mice. Nat Neurosci 23.

Schulz JM, Knoflach F, Hernandez M-C, Bischofberger J (2018) Dendrite-targeting interneurons control synaptic NMDA-receptor activation via nonlinear a5-GABAA receptors. Nat Commun 9:3576.

Schulz JM, Knoflach F, Hernandez MC, Bischofberger J (2019) Enhanced dendritic inhibition and impaired NMDAR activation in a mouse model of down syndrome. J Neurosci 39:5210-5221.

Schuman B, Machold RP, Hashikawa Y, Fuzik J, Fishell GJ, Rudy B (2019) Four unique interneuron populations reside in neocortical layer 1. J Neurosci 39:125-139. 
Serwanski DR, Miralles CP, Christie SB, Mehta AK, Li X, Blas AL De (2006) Synaptic and non-synaptic localization of GABA A receptors containing the alpha5 subunit in the rat brain. J Comp Neurol Neurol 499:458-470.

Silberberg G, Markram H (2007) Disynaptic Inhibition between Neocortical Pyramidal Cells Mediated by Martinotti Cells. Neuron 53:735-746.

Sternfeld F, Carling RW, Jelley RA, Ladduwahetty T, Merchant KJ, Moore KW, Reeve AJ, Street L, O'Connor D, Sohal B, Atack JR, Cook S, Seabrook G, Wafford K, Tattersall FD, Collinson N, Dawson GR, Castro JL, MacLeod AM (2004) Selective, Orally Active $\gamma$-Aminobutyric AcidA a5 Receptor Inverse Agonists as Cognition Enhancers. J Med Chem 47:2176-2179.

Taniguchi H, He M, Wu P, Kim S, Paik R, Sugino K, Kvitsani D, Fu Y, Lu J, Lin Y, Miyoshi G, Shima Y, Fishell G, Nelson SB, Huang ZJ (2011) A Resource of Cre Driver Lines for Genetic Targeting of GABAergic Neurons in Cerebral Cortex. Neuron 71:995-1013.

Tran-Van-Minh A, Cazé RD, Abrahamsson T, Cathala L, Gutkin BS, DiGregorio DA (2015) Contribution of sublinear and supralinear dendritic integration to neuronal computations. Front Cell Neurosci 9:67.

Tremblay R, Lee S, Rudy B (2016) GABAergic Interneurons in the Neocortex: From Cellular Properties to Circuits. Neuron 91:260-292.

Walker F, Möck M, Feyerabend M, Guy J, Wagener RJ, Schubert D, Staiger JF, Witte M (2016) Parvalbumin-and vasoactive intestinal polypeptide-expressing neocortical interneurons impose differential inhibition on Martinotti cells. Nat Commun 7.

Wang Y, Toledo-Rodriguez M, Gupta A, Wu C, Silberberg G, Luo J, Markram H (2004) Anatomical, physiological and molecular properties of Martinotti cells in the somatosensory cortex of the juvenile rat. J Physiol 561:65-90.

Wilson NR, Runyan CA, Wang FL, Sur M (2012) Division and subtraction by distinct cortical inhibitory 

networks in vivo. Nature 488:343-348.

Xu H, Jeong HY, Tremblay R, Rudy B (2013) Neocortical Somatostatin-Expressing GABAergic Interneurons Disinhibit the Thalamorecipient Layer 4. Neuron 77:155-167.

Yavorska I, Wehr M (2016) Somatostatin-expressing inhibitory interneurons in cortical circuits. Front Neural Circuits 10:76.

Zanos P, Nelson ME, Highland JN, Krimmel SR, Georgiou P, Gould TD, Thompson SM (2017) A negative allosteric modulator for $\alpha 5$ subunit- containing GABA receptors exerts a rapid and persistent antidepressant-like action without the side effects of the NMDA receptor antagonist ketamine in mice. eNeuro 4:285-301. MC, Bacci A (2020) Alterations of specific cortical GABAergic circuits underlie abnormal network activity in a mouse model of down syndrome. Elife 9:1-54. 